\title{
Endoscopic ultrasound-guided gallbladder drainage by transduodenal lumen-apposing metal stent in a patient with Roux-en-Y reconstruction
}

\author{
Andrea Anderlonia , Alessandro Fugazza ${ }^{a}$, Luca Maroni ${ }^{\mathrm{a}, \mathrm{b}}$, Edoardo Troncone ${ }^{\mathrm{a}, \mathrm{f}}$, Olivia Milanic, \\ Annalisa Cappello ${ }^{a}$, Asma Alkandarid, Alessandro Repicia ${ }^{\mathrm{a} e}$
}

Humanitas Research Hospital, Rozzano, MI, Italy; Università Politecnica delle Marche, Ancona, AN, Italy; IRCCS Fondaziona Ca' Granda, Ospedale Maggiore Policlinico, Milano, MI, Italy; Alamiri Hospital, Kuwait; University of Rome Tor Vegrata, Rome, Italy

\section{Abstract}

Endoscopic ultrasound-guided gallbladder drainage (EUS-GBD) is increasingly recognized as an effective and safe treatment option in patients with acute cholecystitis (AC) who are deemed unfit for cholecystectomy. Recent studies suggest that an endoscopic approach to biliary decompression may reduce patient discomfort and procedure-related costs compared with the standard percutaneous transhepatic GBD, while maintaining high rates of technical and clinical success. Here we present a case of a EUS-GBD in an elderly patient with AC and with altered anatomy due to a previous partial gastrectomy with Roux-en-Y reconstruction for gastric cancer. The linear array echoendoscope was carefully advanced within the afferent limb until EUS gallbladder visualization was achieved. Transmural GBD was then performed by placement of an electrocautery-enhanced lumen-apposing metal stent. If alternative treatments are precluded, EUS-GBD in patients with AC and altered anatomy is a feasible and effective therapeutic option when performed by experienced endoscopists.

Keywords Endoscopic gallbladder drainage, endoscopic ultrasound, altered anatomy, lumen-apposing metal stent

Ann Gastroenterol 2019; 32 (4): 1-3

\section{Introduction}

Acute cholecystitis (AC) is one of the most prevalent gastrointestinal diseases in clinical practice. The optimal

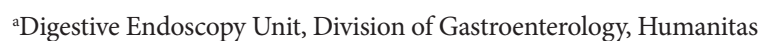
Research Hospital, Rozzano, MI, Italy (Andrea Anderloni, Alessandro Fugazza, Luca Maroni, Edoardo Troncone, Annalisa Cappello, Alessandro Repici); ${ }^{\mathrm{b}}$ Department of Gastroenterology and Hepatology, Università Politecnica delle Marche, Ancona, AN, Italy (Luca Maroni); 'Department of Internal Medicine, IRCCS Fondazione Ca' Granda, Ospedale Maggiore Policlinico, Milano, MI, Italy (Olivia Milani);

'Thanyan Alghanim Center for Gastroenterology and Hematology, Alamiri Hospital, Kuwait (Asma Alkandari); ${ }^{\mathrm{e} H u m a n i t a s}$ University, Rozzano, MI, Italy (Alessandro Repici); fDepartment of System Medicine, University of Rome Tor Vegrata, Rome Italy (Edoardo Troncone

Conflict of Interest: None

Correspondence to: Andrea Anderloni MD, PhD, Digestive Endoscopy Unit, Division of Gastroenterology, Humanitas Research Hospital, Via Manzoni 56, 20089 Rozzano (Milano), Italy,

e-mail: andrea.anderloni@humanitas.it

Received 13 March 2019; accepted 18 April 2019; published online 9 May 2019

Informed consent was obtained from the patient for the publication of information and imaging of the present manuscript and video

DOI: https://doi.org/10.20524/aog.2019.0384 treatment is well established and is based on initial supportive care, antimicrobial therapy, and early cholecystectomy [1]. However, given the advanced age of many patients and the presence of multiple comorbidities, many subjects are deemed unfit for surgery and urgent non-surgical gallbladder drainage (GBD) is usually preferred. [2]. Recently, the feasibility, efficacy and safety of endoscopic ultrasound (EUS)-guided GBD have been confirmed in a systematic review, with an overall technical success rate of $95.8 \%$ and a clinical success rate of 93.4\% [3]. EUS-GBD is, however, traditionally considered technically unfeasible in patients with altered anatomy following surgical resection of the upper gastrointestinal tract. Here we present a case of EUS-GBD by transmural placement of a lumen-apposing metal stent (LAMS) in a patient with Roux-en-Y reconstruction. To the best of our knowledge this is the first such case to be described in the literature.

\section{Case report}

An 86-year-old woman with previous partial gastrectomy and Roux-en-Y reconstruction for gastric cancer presented with AC and cholangitis without clinical response to medical treatment. Because of her multiple comorbidities, the patient 
was deemed unfit for cholecystectomy. Transpapillary GBD by double-balloon enteroscopy was attempted in a referral hospital, but was unsuccessful. Percutaneous transhepatic GBD (PTGBD) was not considered because of concern over complications and the patient's quality of life [4]. The patient was therefore referred to our Unit to attempt an EUS-GBD. EUS evaluation through the gastric remnant demonstrated normal intrahepatic bile ducts and failed to visualize the gallbladder. The linear array echoendoscope was therefore gently advanced under endoscopic and radiologic control as far as the enteroenteric anastomosis, then further on within the afferent limb until the gallbladder was located ultrasonographically. To facilitate progression, the echoendoscope was advanced over a 0.035 -inch guidewire that was pushed forward under fluoroscopic visualization. Transmural gallbladder access was gained with a $19 \mathrm{G}$ needle and bile was aspirated to confirm the correct position. A cholecystogram was then performed and a 0.035 -inch guidewire was coiled in the lumen under fluoroscopic control. Under EUS guidance and fluoroscopic assistance, over-the-wire GBD was then performed by transmural placement of an electrocautery-enhanced (EC)LAMS (Hot Axios, $8 \times 8 \mathrm{~mm}$, Boston Scientific, MA, USA; Fig. 1,2). The following day, a control computed tomography scan documented pneumobilia and confirmed an adequate stent position (Fig. 3). Laboratory findings and symptoms promptly resolved. No adverse events occurred either during the procedure or during the subsequent follow up, extended until 3 months.

\section{Discussion}

In conjunction with medical treatment, early cholecystectomy should ideally be offered to patients suffering from AC. However, in elderly and high-risk subjects with multiple comorbidities, the correct management of the disease may still be challenging [1]. In such patients, cholecystectomy is often contraindicated and their management relies on non-surgical gallbladder decompression. Cholecystostomy using PTGBD access has been extensively used to treat these patients. This approach is relatively easy under transabdominal ultrasound guidance and success rates of PTGBD are high, ranging from $95-100 \%$ [5]. In clinical practice, however, the main concerns for clinicians are the long-term management of external drainage, with a risk of tube displacement/occlusion and infections, especially in elderly patients, and the impact on quality of life. Patient consent may in fact be difficult to obtain in some instances [4]. In recent years, EUS-GBD has emerged as a safe and effective endoscopic therapy. A variety of stents have been employed to achieve gallbladder decompression, ranging from plastic stents, fully-covered self-expandable metal stents (SEMS) to newer LAMS. A recent systematic review showed an overall high technical and clinical success rate when evaluating all available stents. Interestingly, the frequency of adverse events was considerably lower with LAMS than other stents $(9.9 \%$ vs. $18.2 \%$ for plastic stents and $12.3 \%$ for SEMS) [3]. The introduction of a LAMS mounted on an
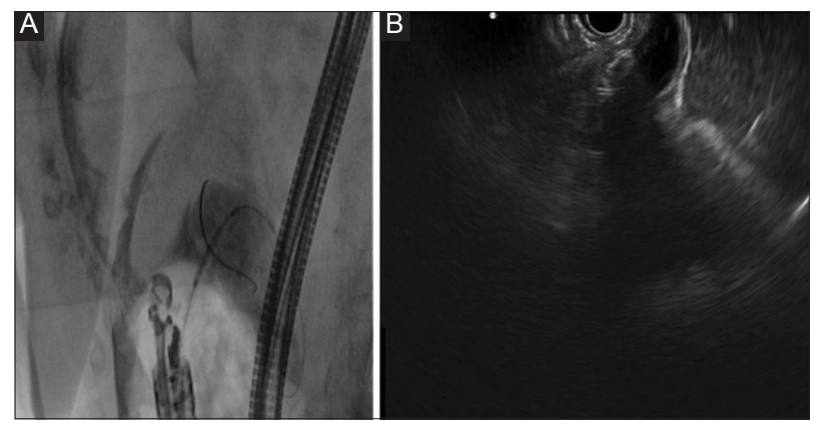

Figure 1 (A) Electrocautery-enhanced advancement of the lumen apposing metal stent (EC-LAMS) under radiological guidance before deployment of the first flange. (B) Trans-duodenal endoscopic ultrasound view of the first flange deployment of the EC-LAMS in the gallbladder lumen

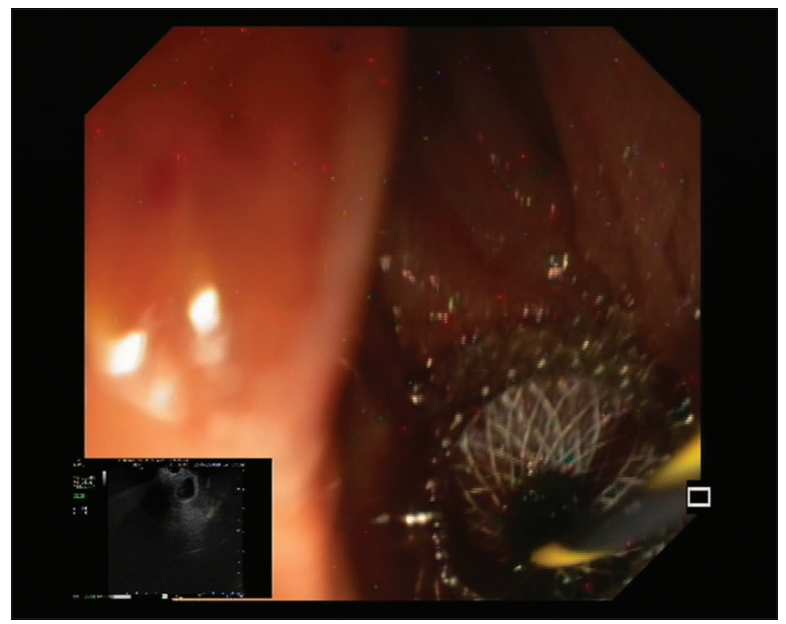

Figure 2 Final endoscopic view of the second flange of the electrocautery-enhanced lumen apposing metal stent in the duodenum after complete release of the stent

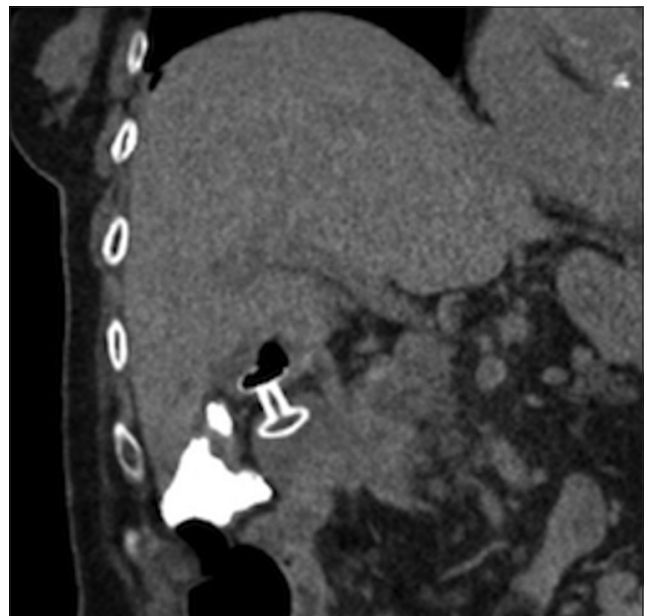

Figure 3 Control computed tomography scan documenting the correct positioning of the electrocautery-enhanced lumen apposing metal stent between the duodenal and the gallbladder lumen, partially filled with air and previously injected contrast agent

EC delivery system (EC-LAMS; Hot Axios, Boston Scientific) allows direct transmural access to the target cavity, without 


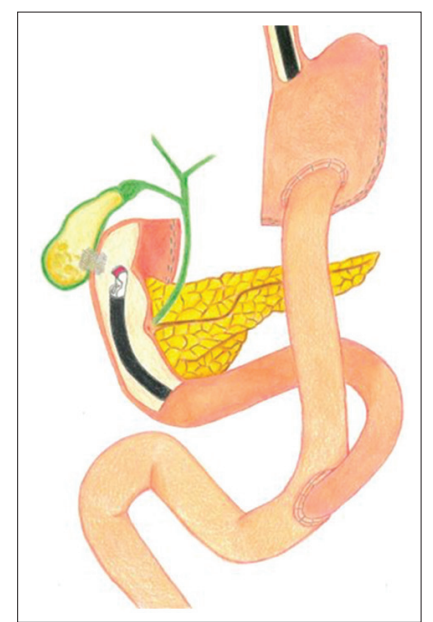

Figure 4 Graphical representation of trans-duodenal endoscopic ultrasound-guided gallbladder drainage in a patient with a Roux-en-Y reconstruction after partial gastrectomy

the need for tract dilatation, reducing the complexity and time of the procedure and possibly improving the outcome. A retrospective multicentric study showed a technical and clinical success of $98.7 \%$ and $95.9 \%$, respectively, when ECLAMS were used in high surgical risk patients [6]. A few studies have compared the outcomes of PTGBD and EUS-GBD. Teoh et al showed comparable rates of technical and clinical success, with significantly fewer adverse events in patients treated with EUS-GBD versus PTGBD (32.2\% vs. 74.6\%) [7]. Similar results in terms of efficacy were demonstrated in 2 other retrospective multicentric studies. Moreover, the length of hospital stay, pain scores and the number of repeated interventions were significantly lower in the EUS-GBD group than in the PTGBD group ( $10 \%$ vs. $24 \%$ ), with important implications for patient morbidity and procedure-related costs $[4,8]$. Finally, EUSGBD with EC-LAMS has also been described in patients with coagulopathy or anticoagulation therapy without major postprocedural bleeding [10]. The long-term safety of EUSGBD appears good; dwell time for GBD has been reported to be up to 364 days without stent-related adverse events for LAMS and up to 3 years for SEMS [9].

To our knowledge, this is the first description of transmural GBD for AC by EC-LAMS in a patient with altered digestive anatomy (Fig. 4). Biliary drainage in patients with a Rouxen-Y reconstruction remains a challenging approach that should be attempted only by experienced endoscopists, once alternative options are excluded based on clinical judgment. In particular, the risks of stent misplacement due to suboptimal scope positioning and bowel injury due to scope progression need to be carefully weighed in each patient. Forward viewing echoendoscopes might allow better advancement and scope positioning in the near future.

EUS-GBD with EC-LAMS is a feasible and effective therapeutic option for AC in patients unfit for surgery. In such patients, this approach may also be suitable to gain anterograde access to the common bile duct for possible endoscopic interventions.

\section{References}

1. Okamoto K, Suzuki K, Takada T, et al. Tokyo Guidelines 2018: flowchart for the management of acute cholecystitis. J Hepatobiliary Pancreat Sci 2018;25:55-72.

2. Baron TH, Grimm IS, Swanstrom LL. Interventional approaches to gallbladder disease. N Engl J Med 2015;373:357-365.

3. Anderloni A, Buda A, Vieceli F, Khashab MA, Hassan C, Repici A. Endoscopic ultrasound-guided transmural stenting for gallbladder drainage in high-risk patients with acute cholecystitis: a systematic review and pooled analysis. Surg Endosc 2016;30:5200-5208.

4. Tyberg A, Saumoy M, Sequeiros EV, et al. EUS-guided versus percutaneous gallbladder drainage: isn't it time to convert? J Clin Gastroenterol 2018;52:79-84.

5. Yeo CS, Tay VW, Low JK, Woon WW, Punamiya SJ, Shelat VG. Outcomes of percutaneous cholecystostomy and predictors of eventual cholecystectomy. J Hepatobiliary Pancreat Sci 2016;23:65-73.

6. Dollhopf M, Larghi A, Will U, et al. EUS-guided gallbladder drainage in patients with acute cholecystitis and high surgical risk using an electrocautery-enhanced lumen-apposing metal stent device. Gastrointest Endosc 2017;86:636-643.

7. Teoh AYB, Serna C, Penas I, et al. Endoscopic ultrasoundguided gallbladder drainage reduces adverse events compared with percutaneous cholecystostomy in patients who are unfit for cholecystectomy. Endoscopy 2017;49:130-138.

8. Irani S, Ngamruengphong S, Teoh A, et al. Similar efficacies of endoscopic ultrasound gallbladder drainage with a lumen-apposing metal stent versus percutaneous transhepatic gallbladder drainage for acute cholecystitis. Clin Gastroenerol Hepatol 2017;15:738-745.

9. Saumoy M, Novikov A, Kahaleh M. Long-term outcomes after EUSguided gallbladder drainage. Endosc Ultrasound 2018;7:97-101.

10. Anderloni A, Attili F, Sferrazza A, et al. EUS-guided gallbladder drainage using a lumen-apposing self-expandable metal stent in patients with coagulopathy or anticoagulation therapy: a case series. Endosc Int Open 2017;5:E1100-E1103. 